\title{
About the Opportunity of Gravitational Radiation Quantum - Graviton Registration
}

\author{
Andrey Nikolaevich Volobuev \\ Department of Physics, Samara State Medical University, Samara, Russia \\ Email address: \\ volobuev47@yandex.ru \\ To cite this article: \\ Andrey Nikolaevich Volobuev. About the Opportunity of Gravitational Radiation Quantum - Graviton Registration. American Journal of \\ Astronomy and Astrophysics. Vol. 6, No. 1, 2018, pp. 1-8. doi: 10.11648/j.ajaa.20180601.11
}

Received: December 30, 2017; Accepted: January 29, 2018; Published: February 28, 2018

\begin{abstract}
Change of a photon frequency in a constant gravitational field is examined. On the basis of Einstein's equation a principle of the gravitational waves occurrence is investigated. The quantum form of an energy-impulse tensor is submitted. Einstein's equation for gravitons is found. It is shown that the solution of this equation for the certain direction represents the sum of a gravitational wave and graviton. It is shown that at approach of graviton to the massive cosmic body radiating gravitational waves there is a resonant pumping of this body energy through gravitational waves in the graviton. It enables for registration of gravitons with the help of the detector which is taking place near to a massive body.
\end{abstract}

Keywords: Gravitational Waves, An Energy-Impulse Tensor, Einstein's Equation, Graviton, A Massive Body, A Resonance, Registration of Gravitons

\section{Introduction}

The modern theory of gravitation - the theory general relativity of Einstein - is a basis for calculation of the astrophysics phenomena. It is generalization Newtonian dynamics, including the law of universal gravitation. As well as Newtonian dynamics the theory general relativity is not the quantum theory. The Einstein's equation for a gravitational field does not have stochastic nature. It contradicts modern physics. For example, for an electron cooperating with a gravitational field with help the Einstein's equation it is possible to calculate position absolutely precisely that contradicts a principle of Heisenberg's uncertainty.

Obviously such situation is unacceptable however the problem of a gravitational field quantization till now is solved though for the solving of this problem many efforts have been applied [1 - 7].

Recently the problem of gravitational waves detecting which description is possible with the help of the Einstein's equation [8 - 12] is solved. It is one more practical confirmation of the general relativity theory correctness.

From the physical point of view the general relativity theory assumes that the mass curvatures a space - time. This curvature of space - time influences all particles moving in space, including and what create a curvature. Influence is carried out and on massless particles. It is connected to a curvature of geodetic lines of space - time on which photons move. A curvature Space - time curvature in the general relativity theory identify with occurrence of some gravitational field due to which there is an interaction of mass particles. Photons also change the frequency in a gravitational field.

Gravitational waves are propagating oscillations of the curved space - time as the similar waves on a water surface there are propagation on a surface of the water particles oscillations.

However in the submitted physical picture of gravitation there is no the major element - quantization of a gravitational field.

Assuming as a whole correctness of the Einstein's equation for a gravitational field we research their some feature prohibiting to quantization of gravitational waves, and hence their incomplete adequacy to a physical picture of the world. 


\section{Photon in Constant Homogeneous Gravitational Field}

For the beginning we shall consider how frequency of electromagnetic radiation quantum (photon) in a constant homogeneous gravitational field changes.

Research we shall carry out in flat space - time the name Minkowski's space. The interval in inertial reference system looks like [13]:

$$
\begin{aligned}
& d s^{2}=c^{2} d \tau^{2}-d X^{2}-d Y^{2}-d Z^{2}= \\
& =g_{00}\left(d X^{0}\right)^{2}-g_{11}\left(d X^{1}\right)^{2}-g_{22}\left(d X^{2}\right)^{2}-g_{33}\left(d X^{3}\right)^{2}
\end{aligned}
$$

where designations there are $X=X^{1}, Y=X^{2}, Z=X^{3}$ - the Cartesian coordinates, $c-$ a light velocity in vacuum, $d \tau$ an interval proper time between events [13], so $c d \tau=X^{0}$, $g_{00}=1, g_{11}=g_{22}=g_{33}=-1$ - metric tensor components:

$$
g_{i k}=\left(\begin{array}{cccc}
1 & 0 & 0 & 0 \\
0 & -1 & 0 & 0 \\
0 & 0 & -1 & 0 \\
0 & 0 & 0 & -1
\end{array}\right)
$$

which signature $(+,-,-,-)$.

From (1) follows:

$$
d \tau=\frac{1}{c} \sqrt{g_{00}} d X^{0}
$$

As it will be shown further in a gravitational field $g_{00}<1$. Therefore, proper (or own) time flows that more slowly than is less $g_{00}$ in the given point of space ( $\Delta \tau=\tau_{2}-\tau_{1}$ decreases; $\tau_{2}$ in field it is less $\tau_{2}$ outside of field). The clock per a gravitational field is slow.

Lagrangian of a gravitational field looks like [13]:

$$
l=T-U=-m c^{2} \sqrt{1-\frac{V^{2}}{c^{2}}}-m \phi_{g}=-m c^{2}\left(1-\frac{1}{2} \frac{V^{2}}{c^{2}}\right)-m \phi_{g}
$$

where $m$ there is mass of a particle in a field, $V$ - its velocity, $\phi_{g}$ - gravitational potential of a field, so acceleration $\dot{\mathbf{V}}=-\operatorname{grad} \phi_{g}$.

Action for a particle in a gravitational field is equal [13]:

$$
S=\int l d \tau=-m c \int\left(c-\frac{V^{2}}{2 c}+\frac{\phi_{g}}{c}\right) d \tau=-m c \int d s
$$

where $S=-m c \int d s$ there is the action for a free material particle.

Hence, the square of the interval $d s$ is equal:

$$
\begin{aligned}
& d s^{2}=\left(c-\frac{V^{2}}{2 c}+\frac{\phi_{g}}{c}\right)^{2} d \tau^{2}= \\
& =\left(c^{2}+\frac{V^{4}}{4 c^{2}}+\frac{\phi_{g}{ }^{2}}{c^{2}}-2 c \frac{V^{2}}{2 c}+2 c \frac{\phi_{g}}{c}-2 \frac{V^{2}}{2 c} \frac{\phi_{g}}{c}\right) d \tau^{2}= \\
& =\left(c^{2}-V^{2}+\frac{\phi_{g}^{2}}{c^{2}}+2 \phi_{g}\right) d \tau^{2}=\left(c^{2}+2 \phi_{g}+\frac{\phi_{g}^{2}}{c^{2}}\right) d \tau^{2}-d \mathbf{r}^{2}= \\
& =c^{2}\left(1+2 \frac{\phi_{g}}{c^{2}}+\frac{\phi_{g}{ }^{2}}{c^{4}}\right) d \tau^{2}-d \mathbf{r}^{2}=c^{2}\left(1+\frac{\phi_{g}}{c^{2}}\right)^{2} d \tau^{2}-d \mathbf{r}^{2}
\end{aligned}
$$

where it is used $d \mathbf{r}=\mathbf{V} d \tau$.

Thus according to (1) components of metric tensor $g_{00}$ in a gravitational field decreases:

$$
g_{00}=\left(1+\frac{\phi_{g}}{c^{2}}\right)^{2} \text { or } \sqrt{g_{00}}=1+\frac{\phi_{g}}{c^{2}}
$$

where $\phi_{g}$ there is negative size.

For the further analysis we use concept of eykonal [13]. Eykonal there is a phase of the periodic function describing a field of electromagnetic wave:

$$
\varphi=\mathbf{k q}-\delta \tau
$$

where $k$ there is a wave vector of eykonal, $q$ - a coordinate vector of eykonal (it is optional Cartesian), $\delta$ - cyclic frequency of eykonal.

Taking into account (3) and (8) it is possible to find frequency eykonal (a photon frequency in the given point in a proper time):

$$
\delta=-\frac{\partial \varphi}{\partial \tau}=-\frac{\partial \varphi}{\partial X^{0}} \frac{\partial X^{0}}{\partial \tau}=-\frac{c}{\sqrt{g_{00}}} \frac{\partial \varphi}{\partial X^{0}}
$$

If to use the world time $t$ (outside of a gravitational field), so that $t=\frac{X^{0}}{c}$ the photon cyclic frequency measured in world time is equal $\delta_{0}=-\frac{\partial \varphi}{\partial t}=-c \frac{\partial \varphi}{\partial X^{0}}$. Hence, according to (9) with the account (7) we have:

$$
\delta=\frac{\delta_{0}}{\sqrt{g_{00}}}=\frac{\delta_{0}}{1+\frac{\phi_{g}}{c^{2}}}
$$

where $\delta_{0}$ there is photon frequency at absence of a gravitational field.

Thus, photon frequency depends on size of a gravitational field potential. As the gravitational field potential it is negative size at approach to the creating a field bodies, the photon frequency $\delta$ grows, and at removal falls (red displacement). For example, for a body in mass $m$ the potential of a field depends on radius $r$ under the formula 
$\phi_{g}=-\frac{\mathrm{km}}{r}$, where $k=6,67 \cdot 10^{-8} \frac{\mathrm{cm}^{3}}{\mathrm{~g} \cdot \mathrm{c}^{2}}$ is a gravitational constant [13].

The eykonal wave vector (or a photon wave vector) $\mathbf{k}=\frac{\partial \varphi}{\partial \mathbf{q}}$, and 4-impulse in the Cartesian coordinates is equal $k_{i}=-\frac{\partial \varphi}{\partial X_{i}} \quad[13]$. But for the 4-impulse the formula $k_{i} k^{i}=k^{0} k^{0}-\mathbf{k k}=0$ is correct. Hence, $\frac{\partial \varphi}{\partial X_{i}} \frac{\partial \varphi}{\partial X^{i}}=0$ there is the eykonal equation.

Let's note that the eykonal is a quantized size. The eykonal

$$
R_{i k l m}=\frac{1}{2}\left(\frac{\partial^{2} g_{i m}}{\partial X^{k} \partial X^{l}}+\frac{\partial^{2} g_{k l}}{\partial X^{i} \partial X^{m}}-\frac{\partial^{2} g_{k m}}{\partial X^{i} \partial X^{l}}-\frac{\partial^{2} g_{i l}}{\partial X^{k} \partial X^{m}}\right)+g_{n p}\left(\Gamma_{k l}^{n} \Gamma_{i m}^{p}-\Gamma_{k m}^{n} \Gamma_{i l}^{p}\right)
$$

where $\Gamma_{k l}^{i}$ there is a projection of a derivative unit vector $\mathbf{e}_{k}$ on coordinate $X^{l}$ on a coordinate axis $X^{i}$ - Cristoffel's symbol $\Gamma_{k l}^{i} e_{i}=\frac{\partial e_{k}}{\partial X^{l}}$ or through of metric tensor $\Gamma_{k l}^{i}=\frac{1}{2} g^{i n}\left(\frac{\partial g_{n k}}{\partial X^{l}}+\frac{\partial g_{n l}}{\partial X^{k}}-\frac{\partial g_{k l}}{\partial X^{n}}\right)$. All indexes, bottom and top, accept values 0 (a time index), 1, 2, 3 (coordinate indexes). As it is usual summation is carried out on indexes repeating in products.

The curvature tensor of space - time it is defined by speed and rapidity of metric tensor change $g_{i k}$ in space - time. Generally there is 10 component of metric tensor - 4 - with identical indexes $(00,11,22,33)$ and 6 with different indexes $\left(01,02,03,12,13,23, C_{4}^{2}=\frac{4 \cdot 3}{1 \cdot 2}=6\right)$, also $g_{i k}=g_{k i}$.

The curvature tensor is the fourth rank. Physical curvature of space - time can be described only the second-rank tensor since the tensor of an energy-impulse creating this curvature is the second-rank tensor. We shall pass in (12) to the secondrank tensor (Ricci's tensor):

$$
R_{i k}=g^{l m} R_{\text {limk }}
$$

Further we shall enter scalar curvature of space under the formula making spur of Ricci's tensor:

$$
R=g^{i k} R_{i k}
$$

The equation for the gravitational field suggested by Einstein look like:

$$
R_{i k}-\frac{1}{2} g_{i k} R=\frac{8 \pi k}{c^{4}} T_{i k}
$$

where quantum is equal:

$$
s_{0}=\hbar \varphi
$$

\section{Occurrence of Gravitational Waves}

Let's consider how Einstein's equation explains gravitational waves.

For writing of the Einstein's equation of a gravitational field it is necessary, first of all, to describe mathematical the curvature of space - time. For the description of mathematical curvature of space it is used the curvature tensor (Riemann's tensor) [13]:

$$
T_{i k}=\left(\begin{array}{cccc}
T_{00} & T_{01} & T_{02} & T_{03} \\
T_{10} & T_{11} & T_{12} & T_{13} \\
T_{20} & T_{21} & T_{22} & T_{23} \\
T_{30} & T_{31} & T_{32} & T_{33}
\end{array}\right)
$$

there is energy-impulse tensor of the second rank the describing source creating curvature of space - time.

Let's multiply the equation (15) at the left on metric tensor $g^{i k}$ :

$$
g^{i k} R_{i k}-\frac{1}{2} g^{i k} g_{i k} R=\frac{8 \pi k}{c^{4}} g^{i k} T_{i k}
$$

Taking into account $R=g^{i k} R_{i k}$ and $g^{i k} g_{i k}=4$ (in fourdimensional space) we shall find:

$$
-R=\frac{8 \pi k}{c^{4}} \mathrm{Sp} T_{i k}=\frac{8 \pi k}{c^{4}} T
$$

where it is designated $\operatorname{Sp} T_{i k}=T$.

Having substituted (18) in (15), we shall find:

$$
R_{i k}=\frac{8 \pi k}{c^{4}}\left(T_{i k}-\frac{1}{2} g_{i k} T\right)
$$

Let's assume that the sources creating a gravitational field are absent, i.e. $T_{i k}=T=0$. In this case according to (19):

$$
R_{i k}=0
$$

If the sources creating a gravitational field are absent the space - time is plane but in it gravitational waves can be propagated. Hence, the equation (20) is the equation of gravitational waves. Similarly at absence in space of sources of an electromagnetic field (moving charges and currents) in it electromagnetic waves can be propagated.

Let's assume further that gravitational waves are connected 
to small change of metric tensor. In this case it is possible to write down [13]:

$$
g_{i k}=g_{i k}^{(0)}+h_{i k}
$$

where $h_{i k}$ there is small gravitational additive to the metric tensor $g_{i k}^{(0)} \gg h_{i k}$.

Substituting (21) in (12) and neglecting for the small gravitational additive squares of Cristoffel's symbols we shall find:

$$
R_{i k l m}=\frac{1}{2}\left(\frac{\partial^{2} h_{i m}}{\partial X^{k} \partial X^{l}}+\frac{\partial^{2} h_{k l}}{\partial X^{i} \partial X^{m}}-\frac{\partial^{2} h_{k m}}{\partial X^{i} \partial X^{l}}-\frac{\partial^{2} h_{i l}}{\partial X^{k} \partial X^{m}}\right)
$$

Let's pass in (22) to Ricci's tensor multiplying (22) at the left on unperturbed metric tensor $g^{(0) l m}$. Thus for contravariant components as against (21) it is necessary to write down:

$$
g^{l m}=g^{(0) l m}-h^{l m}
$$

Thus we shall find:

$$
R_{i k}=\frac{1}{2}\left(-g^{(0) l m} \frac{\partial^{2} h_{i k}}{\partial X^{l} \partial X^{m}}+\frac{\partial^{2} h_{i}^{l}}{\partial X^{k} \partial X^{l}}+\frac{\partial^{2} h_{k}^{l}}{\partial X^{i} \partial X^{l}}-\frac{\partial^{2} h}{\partial X^{i} \partial X^{k}}\right)
$$

where it is designated $h=h_{i}^{i}$. The rise of indexes was carried out by a rule $h_{i}^{k}=g^{(0) k l} h_{i l}$.

Imposing on (24) additional a gauge condition [14]:

$$
\frac{\partial^{2} h_{i}^{l}}{\partial X^{k} \partial X^{l}}+\frac{\partial^{2} h_{k}^{l}}{\partial X^{i} \partial X^{l}}=\frac{\partial^{2} h}{\partial X^{i} \partial X^{k}}
$$

transform (24) to a kind:

$$
R_{i k}=\frac{1}{2}\left(-g^{(0) l m} \frac{\partial^{2} h_{i k}}{\partial X^{l} \partial X^{m}}\right)=\frac{1}{2}\left(\frac{\partial^{2} h_{i k}}{\partial X_{\alpha} \partial X_{\alpha}}-\frac{1}{c^{2}} \frac{\partial^{2} h_{i k}}{\partial t^{2}}\right)
$$

Taking into account the gravitational waves equation (20) we have:

$$
\frac{\partial^{2} h_{i k}}{\partial X_{\alpha} \partial X_{\alpha}}-\frac{1}{c^{2}} \frac{\partial^{2} h_{i k}}{\partial t^{2}}=0
$$

Thus gravitational waves are the cross-section waves propagating with a light velocity $c$.

\section{Quantization of Gravitational Waves}

Before to pass to the gravitational waves quantization we shall be solve one task. We shall find a gravitational field created any gas in space. For calculation we use the formula (18).

The size $K=k_{1} k_{2}$ refers to as the Gaussian curvature of a 3-dimensional surface, and $k_{1}=\frac{1}{\varsigma_{1}}$ and $k_{2}=\frac{1}{\varsigma_{2}}$ there are curvature in mutually perpendicular directions, $\varsigma_{1}$ and $\varsigma_{2}$ radiuses of curvature in these directions. Scalar curvature $R$ (14) is connected with Gaussian curvature a simple ratio $\frac{1}{2} R=K[13]$.

Hence the equation (18) can be transformed to a kind:

$$
-\frac{2}{\varsigma_{1} \varsigma_{2}}=\frac{8 \pi k}{c^{4}}\left(T_{00}+T_{11}+T_{22}+T_{33}\right)
$$

If a body creating a gravitational field is a gas, $T_{11}=T_{22}=T_{33}=P$ there is pressure in gas, a size $T_{00}=\rho c^{2}$, $\rho$ - a gas density. Neglecting pressure in gas in comparison with a mass component $T_{00}=\rho c^{2}$, we have:

$$
-\frac{1}{\varsigma_{1} \varsigma_{2}}=\frac{4 \pi k}{c^{4}} T_{00}=\frac{4 \pi k \rho}{c^{2}}
$$

Assuming $\varsigma_{1}=\varsigma_{2}$ we shall find:

$$
-\frac{1}{\varsigma_{1}^{2}}=\frac{4 \pi k \rho}{c^{2}}
$$

Using a constant $\frac{4 \pi k}{c^{2}}=0,93 \cdot 10^{-27} \frac{\mathrm{cm}}{\mathrm{g}}$ and accepting gas density, for example, according to air density at a surface of the Earth $\rho=1,3 \cdot 10^{-3} \frac{\mathrm{g}}{\mathrm{cm}^{3}} \quad, \quad$ we find $\left|-\varsigma_{1}^{2}\right|=\frac{4 \pi k \rho}{c^{2}}=1,21 \cdot 10^{-30} \frac{1}{\mathrm{~cm}^{2}}$. Hence the radius of space curvature is equal $\varsigma_{1}=\frac{1}{k_{1}}=0,91 \cdot 10^{15} \mathrm{~cm}=0,91 \cdot 10^{10} \mathrm{~km}$.

In calculation for reception of numerical values it was necessary to use the module of a square of curvature. There is an opportunity that space - time is a pseudosphere for which $K=-\frac{1}{\varsigma_{1}^{2}}$. But we do not examine this opportunity since the pseudosphere has an edge of return [15] it is difficult for them to compare any physical property of space - time.

This circumstance sometimes results to that (15) write down Einstein's equation as [16]:

$$
R_{i k}-\frac{1}{2} g_{i k} R=-\frac{8 \pi k}{c^{4}} T_{i k}
$$

Hence there is some uncertainty of a sign before an energy-impulse tensor. Therefore we shall consider the energy-impulse tensor (16) in more detail.

In energy-impulse enters as making the stresses tensor: 


$$
\sigma_{i k}=\left(\begin{array}{ccc}
T_{11} & T_{12} & T_{13} \\
T_{21} & T_{22} & T_{23} \\
T_{31} & T_{32} & T_{33}
\end{array}\right)=\left(\begin{array}{ccc}
\sigma_{X} & \tau_{X Y} & \tau_{X Z} \\
\tau_{Y X} & \sigma_{Y} & \tau_{Y Z} \\
\tau_{Z X} & \tau_{Z Y} & \sigma_{Z}
\end{array}\right)
$$

where $T_{11}, T_{22}, T_{33}$ there are normal stresses, other components $\sigma_{i k}=T_{i k}$ for $i \neq k$ - tangential stresses. A component $T_{00}=\rho c^{2}$ - volumetric density of energy, $T_{10}, T_{20}, T_{30}$ - components of the impulse density multiplied on $c, T_{01}, T_{02}, T_{03}$ - components of the energy flux density divided on $c$.

In [17] it has been shown that in a fluid and gas also there is a uncertainty of a sign on tangential stresses. Moreover the stresses tensor only approximately describes a stressed state of a fluid and gas. In a fluid and gas the stresses tensor is absent. For calculation of a fluid or gas flux it is necessary to use the vector formula connecting force $d \mathbf{F}$ and fluid velocity $\mathbf{V}$ as [17]:

$$
d \mathbf{F}=\ddot{\eta} d \mathbf{S} \times \operatorname{rot} \mathbf{V},
$$

where $d \mathbf{S}$ there is area of contacting layers in a fluid, $\vec{\eta}$ - viscosity tensor of the second rank, which diagonal components there are molecular viscosity, not diagonal components - turbulent viscosity.

If a flux of fluid or gas occurs in a direction axes $X$ the directions of vectors in the formula (33) are shown on figure 1.

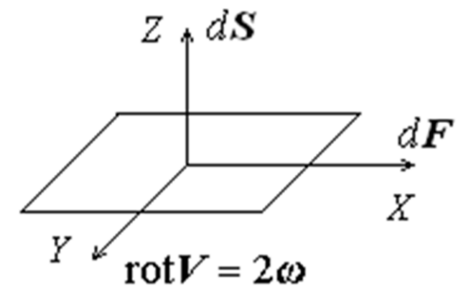

Figure 1. The directions of vectors in the formula (33).

For our purposes the formula (33) using cyclic frequency of a medium rotation $\boldsymbol{\omega}=\frac{1}{2} \operatorname{rot} \mathbf{V}[18]$ it is convenient to write down as:

$$
d \mathbf{F}=2 \vec{\eta} d \mathbf{S} \times \boldsymbol{\omega},
$$

The scalar variant of the formula (34) looks like:

$$
\begin{aligned}
& d F_{X} \mathbf{i}+d F_{Y} \mathbf{j}+d F_{Z} \mathbf{k}=2 \eta\left\{\left(d S_{Y} \omega_{Z}-d S_{Z} \omega_{Y}\right) \mathbf{i}+\left(d S_{Z} \omega_{X}-d S_{X} \omega_{Z}\right) \mathbf{j}+\right. \\
& \left.+\left(d S_{X} \omega_{Y}-d S_{Y} \omega_{X}\right) \mathbf{k}\right\}
\end{aligned}
$$

where $\boldsymbol{i}, \boldsymbol{j}, \boldsymbol{k}$ there are in this case unit vectors of 3 dimensional space. Assuming space homogeneous we use a scalar variant viscosity tensor $\eta$.

At use of the formula (34) the uncertainty of the tangential stresses sign and some other problems connected with stresses tensor [17] on which we shall not stop disappear.

The basic change which we shall introduce to Einstein's equation (15) we shall write down the energy-impulse tensor (16) in the following kind:

$$
T_{i k}=\left(\begin{array}{cccc}
\rho c^{2} & T_{01} & T_{02} & T_{03} \\
T_{10} & \frac{d F_{1}}{d S_{1}} & \frac{d F_{2}}{d S_{1}} & \frac{d F_{3}}{d S_{1}} \\
T_{20} & \frac{d F_{1}}{d S_{2}} & \frac{d F_{2}}{d S_{2}} & \frac{d F_{3}}{d S_{2}} \\
T_{30} & \frac{d F_{1}}{d S_{3}} & \frac{d F_{2}}{d S_{3}} & \frac{d F_{3}}{d S_{3}}
\end{array}\right)
$$

where transition to digital indexes is carried out.

Taking into account (35), we have:

$$
T_{i k}=\left(\begin{array}{cccc}
\rho c^{2} & T_{01} & T_{02} & T_{03} \\
T_{10} & \frac{2 \eta\left(d S_{2} \omega_{3}-d S_{3} \omega_{2}\right)}{d S_{1}} & \frac{2 \eta\left(d S_{3} \omega_{1}-d S_{1} \omega_{3}\right)}{d S_{1}} & \frac{2 \eta\left(d S_{1} \omega_{2}-d S_{2} \omega_{1}\right)}{d S_{1}} \\
T_{20} & \frac{2 \eta\left(d S_{2} \omega_{3}-d S_{3} \omega_{2}\right)}{d S_{2}} & \frac{2 \eta\left(d S_{3} \omega_{1}-d S_{1} \omega_{3}\right)}{d S_{2}} & \frac{2 \eta\left(d S_{1} \omega_{2}-d S_{2} \omega_{1}\right)}{d S_{2}} \\
T_{30} & \frac{2 \eta\left(d S_{2} \omega_{3}-d S_{3} \omega_{2}\right)}{d S_{3}} & \frac{2 \eta\left(d S_{3} \omega_{1}-d S_{1} \omega_{3}\right)}{d S_{3}} & \frac{2 \eta\left(d S_{1} \omega_{2}-d S_{2} \omega_{1}\right)}{d S_{3}}
\end{array}\right) .
$$

The tensor (37) is an energy-impulse tensor causing a curvature of a space - time. Therefore the size $\eta$ should be expressed through the Planck's sizes: Planck's length $\left(\frac{\hbar G}{c^{3}}\right)^{\frac{1}{2}}$, Planck's time $\left(\frac{\hbar G}{c^{5}}\right)^{\frac{1}{2}}$ and Planck's mass $\left(\frac{\hbar c}{G}\right)^{\frac{1}{2}}$.
Instead of size $\eta$ we use it Planck's value $\eta=\frac{\hbar}{\mathrm{V}}$ where $\mathrm{V}$ there is normalizing volume. Besides there are no reasons to assume the areas in (37) various therefore shall accept $d S_{1}=d S_{2}=d S_{3}$. In this case the tensor (37) it will be transformed to a kind: 


$$
T_{i k}=\left(\begin{array}{cccc}
\rho c^{2} & T_{01} & T_{02} & T_{03} \\
T_{10} & \frac{2 \hbar\left(\omega_{3}-\omega_{2}\right)}{\mathrm{V}} & \frac{2 \hbar\left(\omega_{1}-\omega_{3}\right)}{\mathrm{V}} & \frac{2 \hbar\left(\omega_{2}-\omega_{1}\right)}{\mathrm{V}} \\
T_{20} & \frac{2 \hbar\left(\omega_{3}-\omega_{2}\right)}{\mathrm{V}} & \frac{2 \hbar\left(\omega_{1}-\omega_{3}\right)}{\mathrm{V}} & \frac{2 \hbar\left(\omega_{2}-\omega_{1}\right)}{\mathrm{V}} \\
T_{30} & \frac{2 \hbar\left(\omega_{3}-\omega_{2}\right)}{\mathrm{V}} & \frac{2 \hbar\left(\omega_{1}-\omega_{3}\right)}{\mathrm{V}} & \frac{2 \hbar\left(\omega_{2}-\omega_{1}\right)}{\mathrm{V}}
\end{array}\right)
$$

Let's consider for example as looks the energy-impulse tensor at propagation of gravitational radiation to a direction of axis $X_{1}$. Gravitational waves are cross-section hence the vector of frequency is directed along an axis $X_{1}$. In this case the formula (38) looks like:

$$
T_{i k}=\left(\begin{array}{cccc}
\rho c^{2} & T_{01} & T_{02} & T_{03} \\
T_{10} & 0 & \frac{2 \hbar \omega_{1}}{\mathrm{~V}} & -\frac{2 \hbar \omega_{1}}{\mathrm{~V}} \\
T_{20} & 0 & \frac{2 \hbar \omega_{1}}{\mathrm{~V}} & -\frac{2 \hbar \omega_{1}}{\mathrm{~V}} \\
T_{30} & 0 & \frac{2 \hbar \omega_{1}}{\mathrm{~V}} & -\frac{2 \hbar \omega_{1}}{\mathrm{~V}}
\end{array}\right)
$$

Thus in the tensor of energy-impulse the components $\pm \frac{2 \hbar \omega_{1}}{\mathrm{~V}}$ enter which characterize volumetric density of energy of gravitational radiation quantum - graviton. The spin of graviton it is $\pm 2 \hbar$. Two signs of the spin are reflect two directions of polarization: (plus) a vector of cyclic frequency $\omega_{1}$ is directed along direction of a graviton propagation and (minus) against direction of a graviton propagation.

If to accept for example the frequency of background thermal gravitational radiation $\omega_{1}=1,26 \cdot 10^{12} s^{-1}$ [19] then graviton energy is $E=2 \hbar \omega_{1}=2,66 \cdot 10^{-15} \mathrm{erg}=0,00166 \mathrm{eV}$.

In spite of the fact that we have entered the graviton energy in energy-impulse tensor it does not mean that tensor began to have quantum character. Further it is necessary to take into account ideas of the quantum mechanics matrix form and to add at least to tensor components with graviton energy a factor $\exp i\left(r X_{1}-\omega_{1} t\right)$ where $r$ is graviton wave number, $\omega_{1}$ - own graviton frequency. In the matrix form of quantum mechanics $\omega_{1}$ carries the name of spectral frequency and characterizes transition of system from one quantum state in another. The factor $\exp i\left(r X_{1}-\omega_{1} t\right)$ characterizes transition of a gravitational field quantum (graviton) on quantum states in a space - time. We shall note that at record of matrixes in the matrix form of quantum mechanics the exponential factors at a component frequently omit [20]. Thus the energy-impulse tensor will receive a kind:

$$
T_{i k}=\left(\begin{array}{cccc}
\rho c^{2} & T_{01} & T_{02} & T_{03} \\
T_{10} & 0 & \frac{2 \hbar \omega_{1}}{\mathrm{~V}} \exp i\left(r X_{1}-\omega_{1} t\right) & -\frac{2 \hbar \omega_{1}}{\mathrm{~V}} \exp i\left(r X_{1}-\omega_{1} t\right) \\
T_{20} & 0 & \frac{2 \hbar \omega_{1}}{\mathrm{~V}} \exp i\left(r X_{1}-\omega_{1} t\right) & -\frac{2 \hbar \omega_{1}}{\mathrm{~V}} \exp i\left(r X_{1}-\omega_{1} t\right) \\
T_{30} & 0 & \frac{2 \hbar \omega_{1}}{\mathrm{~V}} \exp i\left(r X_{1}-\omega_{1} t\right) & -\frac{2 \hbar \omega_{1}}{\mathrm{~V}} \exp i\left(r X_{1}-\omega_{1} t\right)
\end{array}\right)
$$

The energy-impulse tensor (40) has quantum character.

\section{The Graviton Equation}

For a finding of the graviton equation we shall substitute an energy-impulse tensor in Einstein's equation as (19). The tensor energy-impulse spur (40) looks like $T=\rho c^{2}$. Hence the equation (19) - the graviton equation will be transformed to a kind:

$$
R_{i k}=\frac{8 \pi k}{c^{4}}\left(T_{i k}-\frac{1}{2} g_{i k} \rho c^{2}\right)
$$

Let's consider the graviton equation in almost flat space at absence of a mass component in energy-impulse tensor (40) i.e. at $T=\rho c^{2}=0$. The gravitons practically do not bend space - time. Therefore for the Ricci's tensor in (41) it is possible to use the formula (26):

$$
\frac{\partial^{2} h_{i k}}{\partial X_{1}^{2}}-\frac{1}{c^{2}} \frac{\partial^{2} h_{i k}}{\partial t^{2}}=\frac{16 \pi k}{c^{4}} T_{i k}
$$

The graviton radiation cross-section therefore all components in the equation (42) with indexes 1 at the propagation of graviton in direction $X_{1}$ are excluded. Tensor components $h_{i k}$ remain only: $h_{23}$ and $h_{22}=-h_{33}$ [19]. We shall notice also that in (40) $T_{22}=-T_{33}$.

For a component $h_{23}$ the equation (42) looks like:

$$
\frac{\partial^{2} h_{23}}{\partial X_{1}^{2}}-\frac{1}{c^{2}} \frac{\partial^{2} h_{23}}{\partial t^{2}}=-\frac{16 \pi k}{c^{4}} \frac{2 \hbar \omega}{\mathrm{V}} \exp i\left(r X_{1}-\omega t\right)
$$

For component $h_{22}$ a sign in the right part of the equation is positive. The index 1 at frequency is omitted.

Therefore designating $\chi=h_{i k} \mathrm{~V}$ where $i k=22,23,33$ we shall find:

$$
\frac{\partial^{2} \chi}{\partial X_{1}^{2}}-\frac{1}{c^{2}} \frac{\partial^{2} \chi}{\partial t^{2}}= \pm \frac{32 \pi k \hbar \omega}{c^{4}} \exp i\left(r X_{1}-\omega t\right)
$$

The constant in the right part $\gamma=\frac{32 \pi k \hbar}{c^{4}}=0,87 \cdot 10^{-74} \mathrm{~cm} \cdot s$ is extremely small that reflects practical absence of space - time curvature owing to presence of gravitons.

The equation (44) describes a gravitational wave and graviton propagating from left to right therefore the general 
solution of the equation (44) we shall search as:

$$
\chi=C_{1} f_{1}\left(r X_{1}-\delta t\right)+C_{2} f_{2}(t) \exp i r X_{1},
$$

where $C_{1}$ and $C_{2}$ there are constants, $f_{1}\left(r X_{1}-\delta t\right)$ and $f_{2}(t)$ - any functions, $\delta$ - gravitational wave frequency connected with a velocity of its propagation $r=\frac{\delta}{c}$. Frequency $\delta$ is not equal to own graviton frequency $\omega$. The first term (45) describes the gravitational wave the second term - graviton.

Let's substitute (45) in the equation (44) which have been written down as:

$$
\frac{\partial^{2} \chi}{\partial X_{1}^{2}}-\frac{1}{c^{2}} \frac{\partial^{2} \chi}{\partial t^{2}}= \pm \gamma \omega \exp i\left(r X_{1}-\omega t\right)
$$

The first term (45) satisfies to the equation (46) without the right part. Therefore after substitution we shall find:

$$
\frac{d^{2} f_{2}}{d t^{2}}+\delta^{2} f_{2} \pm \frac{\gamma \omega c^{2}}{C_{2}} \exp (-i \omega t)=0
$$

The particular solution of the equation (47) dependent on own graviton frequency $\omega$ we shall search as:

$$
f_{2}=A \exp (-i \omega t)
$$

Substituting (48) in (47) we shall find:

$$
A= \pm \frac{\gamma \omega c^{2}}{C_{2}\left(\omega^{2}-\delta^{2}\right)}
$$

Hence according to (45) the solution of the equation (44) looks like:

$$
\chi=C_{1} f\left(r X_{1}-\delta t\right) \pm \frac{\gamma \omega c^{2}}{\left(\omega^{2}-\delta^{2}\right)} \exp i\left(r X_{1}-\omega t\right)
$$

Taking into account a designation $\chi=h_{i k} \mathrm{~V}$ where $i k=22,23,33$, and also $\gamma=\frac{32 \pi k \hbar}{c^{4}}$ we shall find:

$$
h_{i k}=\frac{C_{1}}{\mathrm{~V}} f\left(r X_{1}-\delta t\right) \mp \frac{32 \pi k \hbar \omega}{c^{2}\left(\delta^{2}-\omega^{2}\right) \mathrm{V}} \exp i\left(r X_{1}-\omega t\right)
$$

The top sign in (50) and (51) corresponds gravitational additive to the metric tensor $h_{22}$, and the bottom sign $h_{23}$ and $h_{33}$; the first term is responsible for propagation of the gravitational wave the second term characterizes graviton.

\section{Registration of Graviton}

The kind of function (51) allows draw the some conclusions concerning interrelation of a gravitational wave and graviton. Far from massive bodies a graviton as the quantum effect is practically imperceptible. It practically has not influence on registration of the gravitational waves.

However function (51) has interesting feature. At $\omega \rightarrow \delta$ i.e. at aspiration of a graviton frequency to the gravitational wave frequency additional components of the matrix tensor aspire to infinity. The resonance of the graviton and gravitational wave frequencies is observed. This phenomenon can be used for registration of gravitons.

Let's assume that two massive cosmic bodies (for example a double star) rotate around of the common mass centre. This system radiates gravitational waves $h_{i k}=\frac{C_{1}}{\mathrm{~V}} f\left(r X_{1}-\delta t\right)$ with constant frequency $\delta$ [13]. If graviton gets in area of such cosmic bodies its own frequency $\omega$ grows according to the formula similar (10) for photon. According to the formula (51) growth of a graviton energy occurs much faster $2 \hbar \omega$ that specifies resonant pumping of energy of these massive bodies gravitational field (through gravitational radiation) in a graviton. Graviton energy can considerably exceed energy of a gravitational wave and finally graviton can be registered. Probably for this purpose it is necessary to install the detector on massive bodies or near to them (on their planet or the artificial satellite). With the help of such detector it is possible to register abnormal splash in gravitation at graviton passage.

At distance from the bodies a graviton return the energy to a gravitational field of the bodies; its frequency falls (as red displacement in gravitation). Therefore energy of a gravitational field of the massive bodies as a whole does not change at flight near them of graviton. We shall notice also that Riemann's curvature of space - time is positive therefore in the considered effect the additional components of matrix tensor $h_{23}$ and $h_{33}$ take part only. They resonantly grow at flight of a graviton near the massive bodies.

\section{Conclusion}

Use of a vector interrelation between working force and a vector of cyclic frequency of the gravitational field oscillations allows enter the graviton energy $E=2 \hbar \omega$ in energy-impulse tensor which enters into Einstein's equation for a gravitational field. It allows solve the Einstein's equation for the weak gravitational field created graviton only at absence of an external gravitational field.

Power influence of a graviton on a space - time curvature is very insignificant and proportional $\gamma=\frac{32 \pi k \hbar}{c^{4}}=0,87 \cdot 10^{-74} \mathrm{~cm} \cdot \mathrm{s}$.

At approach of a graviton to the massive cosmic bodies (for example to a double star) radiating gravitational waves there is a resonant pumping of the bodies gravitational field energy to a graviton that opens opportunities of its registration. At distance of a graviton from massive bodies its energy is transferred back to a gravitational field of bodies, a frequency of a graviton decreases (red displacement for a 
graviton). Therefore as a whole a graviton has no influence on gravitational field of the bodies.

\section{References}

[1] Bronstein M. P. Quantization of Gravitational Waves. Moscow, JETP, No. 6, 1936, pp. 195-236.

[2] Wheeler J. A. Geons. Phys. Rev. V. 97, 1955, pp. 511-536.

[3] Kiefer C. Quantum Gravity. "New York. Oxford Univ. Press", 2004, 308 p.

[4] Rovelli C. Quantum Gravity. "Cambridge. Cambridge Univ. Press", 2004, 455 p.

[5] Rubakov V. A., Tinyakov P. G. Infrared - modified Gravities and Massive Gravitons. Moscow. Uspekhi Fizicheskikh Nauk. V. 178, No. 8, 2008, pp. 785-822.

[6] Luis Santiago Ridao, Mauricio Bellini. Towards relativistic quantum geometry, Phys. Lett. B751, 2015, pp. 565-571.

[7] Fuminory Hasegawa, Kazunory Nakayama, Takahiro Terada, Yusuke Yamada. Gravitino problem in inflation driven by inflaton-polonyi Kahler coupling, Phys. Lett. B777, 2018, pp. 270-274.

[8] Abbott B. P. et al. Observation of Gravitational Waves from a Binary Black Hole Merger, Phys. Rev. Lett. V. 116, 2016, p. 061102 .

[9] Jinno R. and Takimoto M. Gravitational Waves from Bubble Collisions: An Analytic Derivation, Phys. Rev. D, V. 95, issue 2, 2017, p. 024009.
[10] Capozziello S., Stabile A. Gravitational Waves in Fourth Order gravity, Astrophysics and Space Science, V. 358, 2015, p. 27.

[11] Jesus Martin Romero, Mauricio Bellini, Jose Edgar Madriz Aguilar. Gravitational Waves from a Wely-integrable Maniford: A New Formalism. Physics of the Dark Universe, V. 13, 2016, pp. 1-6.

[12] Amarjit Soni, Yue Zhang. Gravitational Waves from SU(N) Glueball Dark Matter, Phys. Lett. B771, 2017, pp. 379-384.

[13] Landau L. D., Lifshitz E. M. Theory of field. Moscow, "Science", 1967, pp. 173, 292, 301, 317, 336, 337, 344, 402.

[14] Mathematical Physics. The Encyclopedia. Editor Faddeev L. D. Moscow. "Scientific Publishing House "Big Russian Encyclopedia”, 1998, p. 153.

[15] Bakelman I. J. Higher Geometry. Moscow. "Education", 1967, p. 349.

[16] Rashevskij P. K. Riemann's Geometry and Tensor Analysis. Moscow, "Publishing House LKI”, 2008. p. 635.

[17] Volobuev A. N. Basis of Nonsymmetrical Hydromechanics. New York. "Published by Nova Science Publishers, Inc." 2012, pp. 87, 88, 184.

[18] Schlichting H. The Boundary Layer Theory. Moscow. "Science", 1974, p. 63.

[19] Zeldovich J. B., Novikov I. D. The Theory of Gravitation and Evolution of Stars. Moscow. "Science", 1971, p. 55, 103.

[20] Mott N., Sneddon I. Wave Mechanics and its Applications. Moscow. "ComKniga", 2007, p. 389. 